\title{
Laser-induced radial birefringence and spin-to-orbital optical angular momentum conversion in silver-doped glasses
}

\author{
Jafar Mostafavi Amjad, ${ }^{1}$ Hamid Reza Khalesifard, ${ }^{1}$ Sergei Slussarenko, ${ }^{2}$ Ebrahim Karimi, ${ }^{2}$, 国 Lorenzo Marrucci, ${ }^{2,3}$ \\ and Enrico Santamato ${ }^{2}$ \\ 1) Department of Physics, Institute for Advanced Studies in Basic Sciences (IASBS), 45137-66731 Zanjan, \\ Iran \\ ${ }^{2)}$ Dipartimento di Scienze Fisiche, Università di Napoli "Federico II", Complesso Universitario di Monte S. Angelo, \\ 80126 Napoli, Italy \\ ${ }^{3)}$ CNR-SPIN, Complesso di Monte S. Angelo, 80126 Napoli, Italy
}

Samples of $\mathrm{Ag}^{+} / \mathrm{Na}^{+}$ion-exchanged glass that have been subject to intense laser irradiation may develop novel optical properties, as a consequence of the formation of patterns of silver nanoparticles and other structures. Here, we report the observation of a laser-induced permanent transverse birefringence, with the optical axis forming a radial pattern, as revealed by the spin-to-orbital angular momentum conversion occurring in a probe light beam. The birefringence pattern can be modeled well as resulting from thermallyinduced stresses arising in the silver-doped glass during laser exposure, although the actual mechanism leading to the permanent anisotropy is probably more complex.

Metal-doped dielectrics may acquire interesting linear and nonlinear optical properties, arising from the combination of the dielectric transparency and of the metallic surface-plasmon-polariton (SPP) response, particularly when the metallic component aggregates in nanoparticles, clusters, or more complex structures, giving rise to a kind of self-assembled metamaterial. ${ }^{1,2}$ The SPP resonances depend critically on the metal cluster size, shape, and distribution so that these materials can be effectively tailored to make novel functional devices for optoelectronics and telecommunications. ${ }^{3-5}$ Silver-ion-exchanged glass is one of the most promising materials, due to its easy manufacturing and flexible properties. Different techniques have been demonstrated to control the silver cluster structure, size, distribution, and phase separation, most of which are based on applying a strong DC electric field or on exposing the materials to intense laser illumination. $^{2,6-10}$.

Recently, Nahal et al. reported that initially isotropic silver-doped glasses become birefringent under strong laser illumination during preparation, with the optical axis lying along the laser propagation direction. ${ }^{11}$ In this paper, we report the observation that laser exposure generates also a significant transverse birefringence, with the induced optical axis forming a radial pattern around the laser beam axis. This pattern gives rise to optical depolarization effects occurring at small deflection angle (in contrast to the large-angle conoscopic effects reported in Ref. 11). We link these birefringence effects to a partial conversion of the spin angular momentum (SAM) of the incoming photons into orbital angular momentum (OAM), a process known as spin-to-orbital angular momentum conversion (STOC). ${ }^{12,13}$ As a consequence of STOC, the SAM and OAM degrees of freedom of photons become entangled and the polarization becomes spatially variant, giving rise to the apparent depolarization

\footnotetext{
a) Electronic mail: karimi@na.infn.it
}

of the light beam. It should be emphasized however that this form of depolarization is not originated by random dephasing, as in natural light sources, but it is a deterministic phenomenon that preserves the overall light coherence.

The investigated samples were prepared by the well known $\mathrm{Ag}^{+} / \mathrm{Na}^{+}$ion exchange technique. ${ }^{11,14,15}$ Sodalime glass slides of dimensions $39 \times 25 \times 0.85 \mathrm{~mm}$ merged into a 96:4 (weight ratio) molten mixture of $\mathrm{NaNO}_{3}$ and $\mathrm{AgNO}_{3}$ at $400^{\circ} \mathrm{C}$ for $4 \mathrm{hr}$. The chemical composition of the glass was: $\mathrm{SiO}_{2}, 80 \% ; \mathrm{CaO}, 9.41 \% ; \mathrm{Na}_{2} \mathrm{O}$, $4.0 \%$; $\mathrm{MgO}, 3.3 \% ; \mathrm{Al}_{2} \mathrm{O}_{3}, 2.2 \%$; $\mathrm{K}_{2} \mathrm{O}, 0.41 \% ; \mathrm{S}, 0.2 \%$; $\mathrm{Fe}_{2} \mathrm{O}_{3}, 0.11 \% ; \mathrm{P}_{2} \mathrm{O}_{5}, 0.11 \%$; others, $0.26 \%$ (percentages in weight). The ion-exchanged glass samples were then irradiated with a multi-line $\mathrm{Ar}^{+}$laser beam focused onto a spot with a $3 \mathrm{~mm}$ diameter. Three samples were prepared with different exposure doses: $100 \mathrm{~W} / \mathrm{cm}^{2}$ for $1 \mathrm{~s}$ (S1), 30 $\mathrm{W} / \mathrm{cm}^{2}$ for $10 \mathrm{~s}(\mathrm{~S} 2)$, and $100 \mathrm{~W} / \mathrm{cm}^{2}$ for $10 \mathrm{~s}$ (S3). These silver-doped glasses are moderately absorbing in the visible, so the laser beam leads to a strong local heating during exposure and to the development of a radial thermal gradient towards the center of the laser spot. This thermal gradient generates in turn a mechanical stress leading, by elasto-optic effect, to a cylindrically symmetric radial birefringence. In the case of a Gaussian beam profile, the birefringence pattern induced by this mechanism during exposure can be calculated analytically. ${ }^{16,17}$ For an initially isotropic material such as glass, the local direction of the optical axis is radially oriented along the temperature gradient and the local optical birefringent retardation $\delta$ is radially symmetric and given by

$$
\delta(r)=\delta_{0}\left[1+\frac{1}{2}\left(\frac{r_{0}}{r}\right)^{2}\left(e^{-\frac{2 r^{2}}{r_{0}^{2}}}-1\right)\right]
$$

where $r$ is the radial coordinate, $r_{0}$ is the waist radius of the laser beam and $\delta_{0}$ is the asymptotic phase retardation for large radii. The latter will depend on the glass thermal, optical, and elasto-optical properties and on the light power. In our case, the actual mechanism leading 
to the radial birefringence must clearly be more complicated than that described above, because during laser irradiation the $\mathrm{Ag}^{+}$ions are known to form nano-clusters which migrate around the beam axis, contributing to the induced optical anisotropy. In addition, the laser-induced thermal stresses can deform the surface of the glass sample, producing a lensing effect. Once the laser light is turned off and the sample cools down, the silver-particle distribution, stresses, and surface deformation are frozen, and the radial birefringence thus becomes persistent. A detailed model of these rather complex effects is under investigation, but it is reasonable to assume that Eq. (1) remains approximately valid as a phenomenological model, with parameters $\delta_{0}$ and $r_{0}$ now depending also on the laser intensity and exposure time and on the $\mathrm{Ag}^{+}$ion concentration and mobility.

The main optical effect of the birefringence pattern given by Eq. (1) can be simply obtained using the Jones operator, $\widehat{U}$, acting on the light polarization. In the basis of the circular polarizations, the Jones operator $\widehat{U}$ assumes the simple form

$$
\widehat{U} \cdot| \pm\rangle=\cos \left[\frac{\delta(r)}{2}\right]| \pm\rangle-i \sin \left[\frac{\delta(r)}{2}\right]|\mp\rangle e^{ \pm 2 i \phi},
$$

where $|+\rangle$ and $|-\rangle$ denote the left and right circular polarizations, respectively and $\phi$ is the azimuthal angle in the transverse plane. As we see, if the input light is circularly polarized, the transmitted light will be the superposition of an unmodified wave, with amplitude reduced by the factor $\cos (\delta / 2)$, and a new wave having opposite polarization handedness, with amplitude factor $\sin (\delta / 2)$. The latter also exhibits a vortex phase factor $e^{ \pm 2 i \phi}$, corresponding to an OAM of $\pm 2 \hbar$ per photon, exactly balancing the variation of SAM (i.e., from $\pm \hbar$ to $\mp \hbar$ ): this is just the STOC process. ${ }^{12,13}$ In the following, for brevity, the polarization-inverted component with nonzero OAM (assuming that the input has zero OAM) will be referred to as the "STOC component" of the outgoing light.

The polarization of the STOC component is always orthogonal to the polarization of the input beam, which explains the observed depolarization of the transmitted light. Depolarization occurs for any input polarization (linear or elliptical), since any polarization can be decomposed into the left and right circular components, each undergoing STOC to a different (opposite) OAM value, so after STOC they do not add up coherently anymore. The STOC efficiency is given by $\sin ^{2}[\delta(r) / 2]$. Therefore, complete STOC is expected only for certain radii, determined by the Eq. $\delta(r)=(2 n+1) \pi$ with $n$ integer, with partial STOC elsewhere. Because in the present case $\delta(r)$ is not small generally, this varying STOC efficiency should give rise to a radially-oscillating intensity profile of the transmitted STOC beam.

The optical properties acquired by our samples after the laser-induced effects described above have become permanent were studied using a probe laser beam having a gaussian input profile with a beam waist diameter of about $4 \mathrm{~mm}$, for two different input polarizations: left-

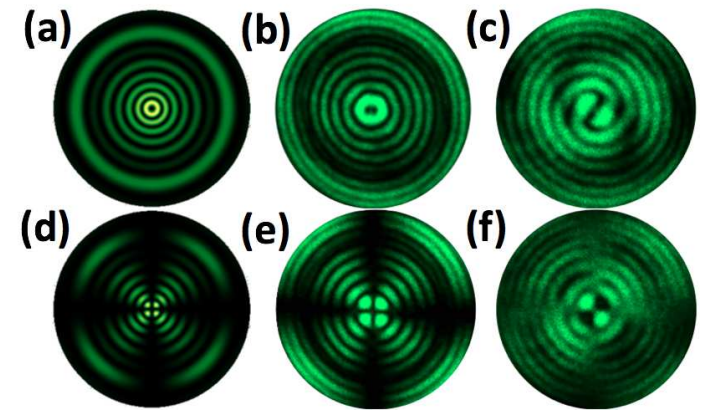

FIG. 1. (Color online) Far-field intensity profiles of a gaussian probe light beam passing through sample S3. Upper panels are for a left-circular input polarization, lower panels for linear polarization (vertical in the figures). Panels (a) and (d) are the profiles calculated from our theory, (b) and (e) are the experimental ones, and (c) and (f) are interference patterns with a gaussian reference beam. In the calculated patterns, we set $\delta_{0}=45$ and $r_{0}=0.7 \mathrm{~mm}$, and assumed a probe beam waist of $2 \mathrm{~mm}$.

circular and linear. In both cases, in transmission we observed a large far-field ring pattern, which is typical of transverse phase-modulation, as observed also by other authors and due to the isotropic laser-induced modulation of optical properties. ${ }^{10}$ The depolarized light, however, appears only in a small circular region around the pattern center, that we selected by using an iris and a crossed polarizer. Figures 1b,e show the experimental intensity patterns of this depolarized light, for two different input polarizations. These patterns are very well reproduced by our calculations of the STOC component based on our model, as shown in Figs. 1a,d. In the case of input circular polarization, in particular, the intensity profile is cylindrically symmetric and presents a dark singularity at the center, characteristic of a beam carrying nonzero OAM. Moreover, the patterns exhibit concentric circular fringes resulting from the radial modulation of STOC efficiency, as discussed above. We also recorded the interference patterns resulting from the superposition of the depolarized light from the samples with a reference gaussian beam (having the same polarization), in order to check the optical phase distribution. The interference pattern obtained for a circular polarization, in particular, presented the characteristic double spiral pattern, confirming that the OAM eigenvalue is $|\ell|=2$, in units of $\hbar^{12}$

We investigated the behavior of the overall STOC efficiency (defined as the power ratio between the spaceintegrated STOC component and the overall transmitted light) for our different samples, having different exposure parameters. Figure 2 shows that the overall STOC efficiency is an increasing function of the light energy dose absorbed by the sample during its preparation, as could be reasonably expected.

The images shown in Fig. 1 provide a first qualitative confirmation of the occurrence of STOC in our samples. A more quantitative analysis can be performed by apply- 


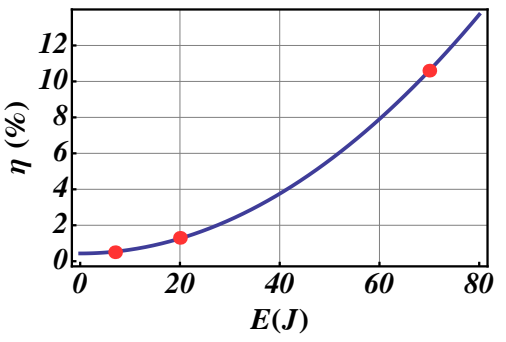

FIG. 2. (Color online) The STOC efficiency as a function of the total light energy deposited during the sample preparation. The circles correspond to the measured efficiency in our three samples S1, S2, and S3. The curve is a best fit with the quadratic expression $\eta(E)=a+b E^{2}$.

ing the methods of quantum tomography to reconstruct the probe photon OAM quantum state at the exit of the sample. This method allows us to retrieve both the amplitudes and relative phases of all OAM components of the transmitted light beam, despite the fact that they do not interfere. Our model predicts that, for any input polarization, only superpositions of OAM eigenstates with $\ell= \pm 2$ should be generated. Therefore, only the twodimensional OAM Hilbert subspace spanned by $\ell= \pm 2$ is relevant. This space is isomorphous to the polarization space, so we may use "Stokes-like" parameters to describe it. The correspondence between the OAM and polarization spaces can be made by associating the OAM eigenstates $\ell= \pm 2$ with the left and right polarization states, respectively, and any superposition of the OAM eigenstates $\ell= \pm 2$ with the corresponding elliptical polarization state. Thus, just as in the case of polarization, to gain full information on the OAM state we need only four independent measurements of the Stokes' parameters $S_{i}$ $(i=0, \ldots, 3)$, from which the full density matrix of the state can be retrieved. ${ }^{18}$ To measure all OAM Stokes-like parameters we used six gray-scale computer-generated holograms (CGH), as reported elsewhere. ${ }^{19}$ These holograms were displayed in turn on a spatial light modulator (SLM) on which the depolarized light from the sample was made to impinge. The resulting far-field intensity in the central area of the first-order diffraction was then measured for each CGH and, by combining these data, all Stokes-like parameters were calculated. ${ }^{19}$ The results of this OAM photon state tomography for the two cases of left-circular and linear input polarization of the probe beam are shown in Fig. 3, which presents the real and imaginary parts of the OAM density matrix. As we see, in the case of the left circular input polarization, all output photons of the depolarized component were put into the $\ell=+2$ OAM eigenstate (confirming again the STOC effect), while in the case of the vertical input polarization we obtained an equal-weight coherent superposition of the two $\ell= \pm 2$ OAM eigenstates. Moreover, the fidelities of these states with the optical-field azimuthal profiles of ideal $|\ell|=2$ and $(|\ell=2\rangle+|\ell=-2\rangle) / \sqrt{2}$ modes were found to be 0.97 and 0.91 , respectively. This confirms
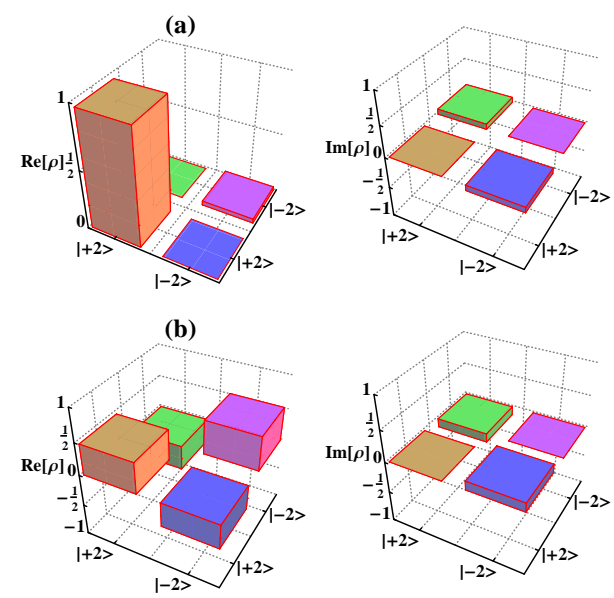

FIG. 3. (Color online) Experimental density matrix for a (a) left-circularly polarized and (b) linearly polarized input probe beam, as emerging from sample S3. Left and right panels show the real and imaginary part of the density matrix, respectively (but, within the experimental errors, all measured density-matrix elements were found to be real).

that the experimental results are in excellent agreement with our STOC-based model.

In conclusion, we have reported the appearance of a radial birefringence pattern in laser-exposed ion-exchanged silver-doped glasses, as demonstrated by the occurrence of spin-to-orbital angular momentum conversion in a probe beam. This pattern presumably arises as a result of laser-induced thermal gradients and ensuing silver nanoparticle migration and permanently induced mechanical stresses in the glass.

We acknowledge the financial support of the FETOpen program within the $7^{\text {th }}$ Framework Programme of the European Commission under grant No. 255914, Phorbitech, and of IASBS research council under the grant No. G2002IASBS101

${ }^{1} \mathrm{U}$. Kreibig and M. Vollmer, Optical properties of metal clusters, Springer series in materials science, Vol. 25 (Springer, Berlin, 1995).

${ }^{2}$ A. Abdolvand, A. Podlipensky, S. Matthias, F. Syrowatka, U. Gösele, G. Seifert, and H. Graener, Advanced Materials, 17, 2983 (2005).

${ }^{3}$ J. Auxier, S. Honkanen, A. Schülzgen, M. Morrell, M. Leigh, S. Sen, N. Borrelli, and N. Peyghambarian, J. of the Opt. Soc. of Am. B, 23, 1037 (2006).

${ }^{4}$ F. Gonella, G. Mattei, P. Mazzoldi, E. Cattaruzza, G. W. Arnold, G. Battaglin, P. Calvelli, R. Polloni, R. Bertoncello, and R. F. Haglund, Appl. Phys. Lett. 69, 3101 (1996).

${ }^{5}$ S. Friberg and P. Smith, IEEE Journal of Quantum Electronics 23, 2089 (1987).

${ }^{6}$ F. Stietz, Appl. Phys. A-Materials Science \& Processing 72, 381 (2001).

${ }^{7}$ R Jin, Y. Cao, E. Hao, G. S. Métraux, G. C. Schatz, and C. A. Mirkin, Nature 425, 487 (2003).

${ }^{8}$ A. Nahal, J. Mostafavi-Amjad, A. Ghods, M. R. H. Khajehpour, S. N. S. Reihani, and M. R. Kolahchi, Journal of Appl. Phys. 100, 1063 (2006).

${ }^{9}$ A. Miotello, Appl. Phys. Lett. 79, 2456 (2001). 
${ }^{10}$ Y. Kaganovskii, I. Antonov, F. Bass, M. Rosenbluh, and A. Lipovskii, Journal of Appl. Phys., 89, 8273 (2001).

${ }^{11} \mathrm{~A}$. Nahal and F. Moslehirad, Journal of Materials Science, 42, 9075 (2007).

${ }^{12}$ L. Marrucci, C. Manzo, and D. Paparo, Phys. Rev. Lett. 96, 163905 (2006).

${ }^{13}$ L. Marrucci, E. Karimi, S. Slussarenko, B. Piccirillo, E. Santamato, E. Nagali, and F. Sciarrino, J. Opt. 13, 064001 (2011).

${ }^{14}$ A. Nahal, H. R. Khalesifard, Opt. Mat. 29, 987, (2007).
${ }^{15}$ S. I. Najafi, Introduction to Glass Integrated Optics, edited by S. I. Najafi (Artech House Publishers,1992).

${ }^{16}$ E. Khazanov, O.V. Kulagin, S. Yoshida, D.B. Tanner, and D.H. Reitze, IEEE Journal of Quantum Electronics 35, 1116 (1999).

${ }^{17}$ S. Mosca, B. Canuel, E. Karimi, B. Piccirillo, L. Marrucci, R. De Rosa, E. Genin, L. Milano, and E. Santamato, Phys. Rev. A 82 , 043806 (2010).

${ }^{18}$ M. Padgett and J. Courtial, Opt. Lett., 24, 430 (1999).

${ }^{19}$ E. Nagali, F. Sciarrino, F. De Martini, B. Piccirillo, E. Karimi, L. Marrucci, and E. Santamato, Opt. Express, 17, 18745 (2009). 